\title{
Planning and Design of Computer Network Management System under the Background of Big Data
}

\author{
Huangying Zha \\ Information and Modern Educational Technology Center, Xi'an University, 710065
}

\begin{abstract}
Keywords: Big Data; Computer Network Management; Design of Computer Network Management System
\end{abstract}

\begin{abstract}
Computer networks have become more and more widely used in every corner of our lives and work. The popularity and application of computers indicates that the era of big data has arrived and plays a very important role in our lives and work. With the widespread use of various application systems in computer network environments, computer networks are becoming larger and larger, and network devices are becoming more diverse. To effectively manage and integrate such a large number of application systems, we are required to have good management tools to ensure the normal operation of the computer network system. This paper introduces the definition and characteristics of network management. By analyzing the composition and architecture of network management system and combining the functions of network management, the model of computer network management system based on big data background is designed.
\end{abstract}

\section{The Status Quo of Computer Network Management System in the Context of Big Data}

The era of big data is a very popular word, so what is big data? Big data refers to a very large number of data set with various types. Volume, Variety, Velocity, and Value are the distinguishing features of Big Data. Figure 1.1 shows the 4V features of big data and the characteristics of each feature:

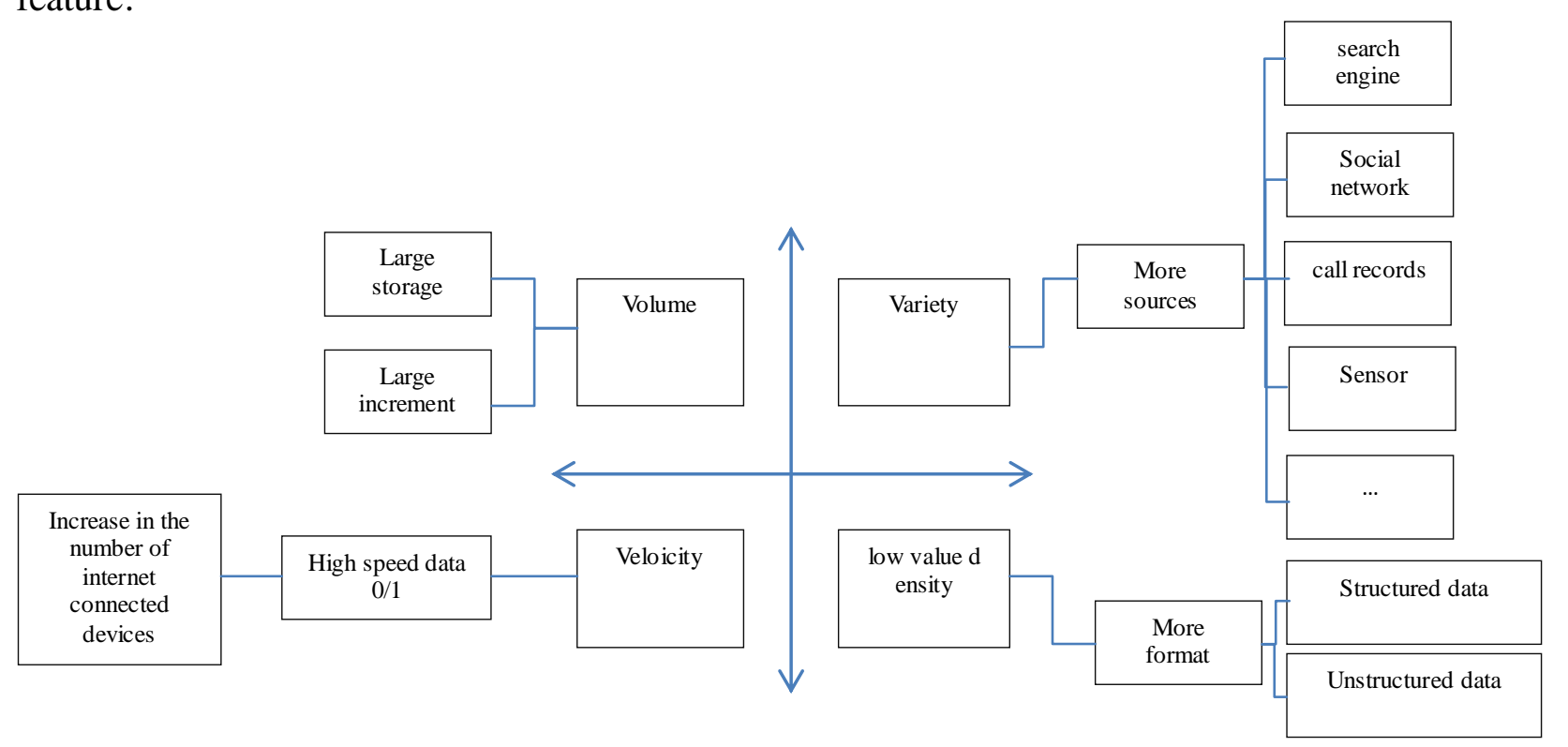

Figure 1.1 4V Features of Big Data and the Characteristics

With the advent of the era of big data, computer network management systems have the following features: (1) In terms of data: the types and quantities of data information in the network are increasing, and the value and meaning of different information in different fields are also significantly different. How can we monitor, screen and utilize these data information, so that the accuracy of automatic collection and judgment of information is greatly improved, and it can also better protect information security and improve network management efficiency. (2) In terms of system: As the network system structure becomes more and more complex, the network load is 
ever-changing, and the systems used in the network are more diverse, making it difficult for the traditional network control theory to make the management of the computer network efficient.

Therefore, in the environment where big data is prevalent, we need to use a more intelligent and efficient network management system to improve the efficiency of information processing and network security.

\section{Design of Computer Network Management System Based on Big Data Background}

Because of the need for further construction in the future, it is difficult to simply integrate and manage existing computer networks and application systems with off-the-shelf network management systems. Therefore, we must design and implement a system based on computer network management under the background of big data according to the needs of existing network resources and application management. At the same time, we must also consider the scalability of the network management system.

The network hardware resources managed by the computer network management system may be physical media and networked devices including physical layer and data link layer, including processors and storage devices, and other computer peripheral devices; The managed network software resources may be, for example, Windows, Linux, and operating system software including distributed applications, communication software capable of implementing communication protocols, and application software.

From the perspective of the composition of the system, the modern network management system consists of four parts: a network management system (NMS) that implements various network management functions on a single or multiple network host nodes, and multiple residency in the network management system. A management agent (Managed Agent) that provides network devices and management system request setting information on the management network device, and a management information base corresponding to the management agent that can dynamically refresh and provide data and storage management agent setting data to the management agent MIB (Management Information Base), a shared data format that defines the communication message format of the network management and the management information base, and a network management protocol that describes the data communication mechanism between the network management system and the management agent. The specific structural relationship is shown in Figure 2.1:

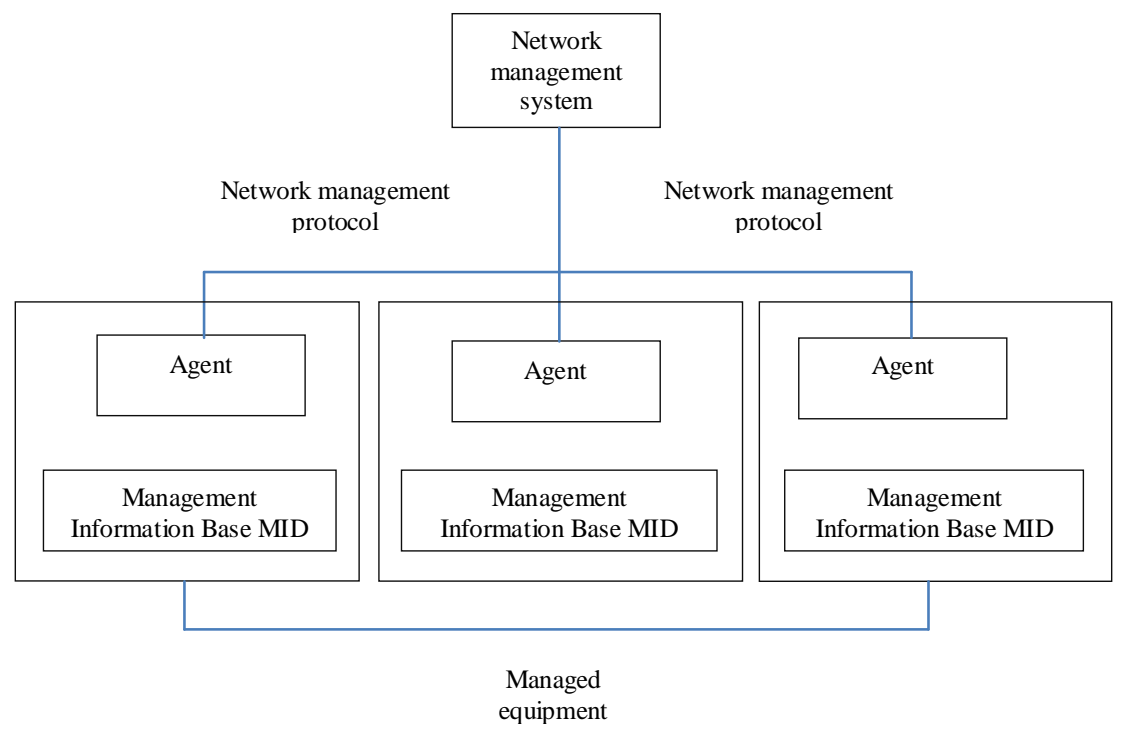

Figure 2.1 The relationship between the basic components of computer network management

The architecture model of the computer network management system includes a sub-model describing four aspects: an information model that is managed and directly related to the management information base MIB, and an organization model describing the processing and 
supporting organization (Organization Model), a Communication Model describing the communication methods and processes required for real-time management purposes, and a Function Model describing the composition of network management tasks.

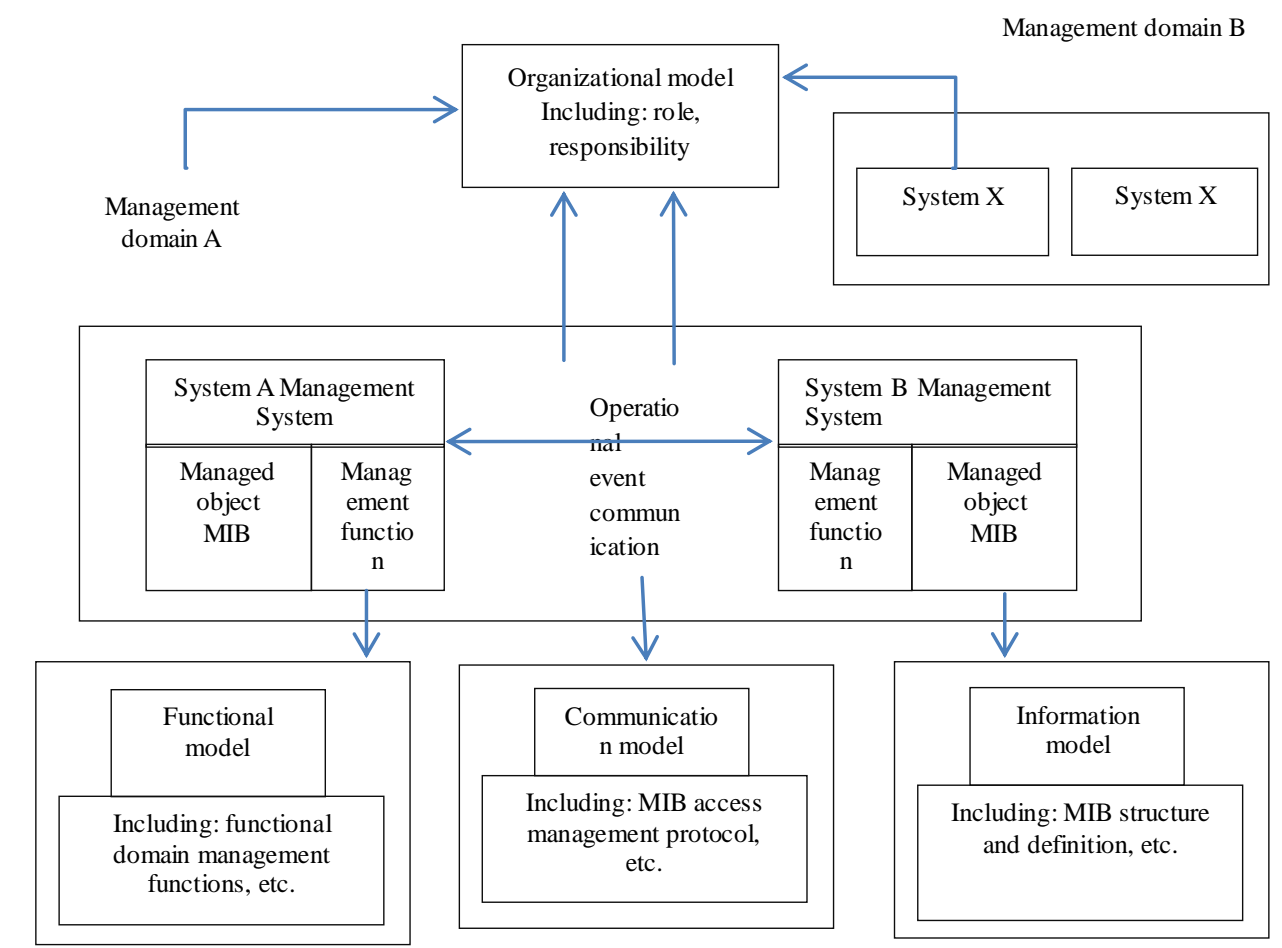

Figure 2.2 Sub-models of computer network management system structure and relationships between them

To establish a friendly computer network management system, the following guidelines should be followed: provide a relational database interface for storing and retrieving the required information; provide a graphical analysis tool that allows administrators to visually observe changes in data; provide network monitoring that enables administrators to grasp the current state of the network and control capabilities that enable administrators to take action to affect the operational status of the network; manage as many network protocols as possible and reduce the system overhead as much as possible under the same network management cost, and improve the operating efficiency of the network; support different network management systems and connect different metropolitan area networks or local area networks to form a unified network management operation mechanism of the whole network; support different network management platforms and adopt standardized network management mechanisms and protocols.

\section{Design of Computer Network Management Function Based on Big Data Background}

The computer network management system structure includes: configuration management that can set and change configuration parameters in the network as a basis of network management; analyzes performance management in the network that has quota problems and monitors network operation performance; ensure that the network can provide continuous and reliable fault management for monitoring and isolating network faults; including physical device security and security management of access rights of network resources; management of billing management for network spending. The functional structure of the computer network management system is shown in Figure 3.1 . 


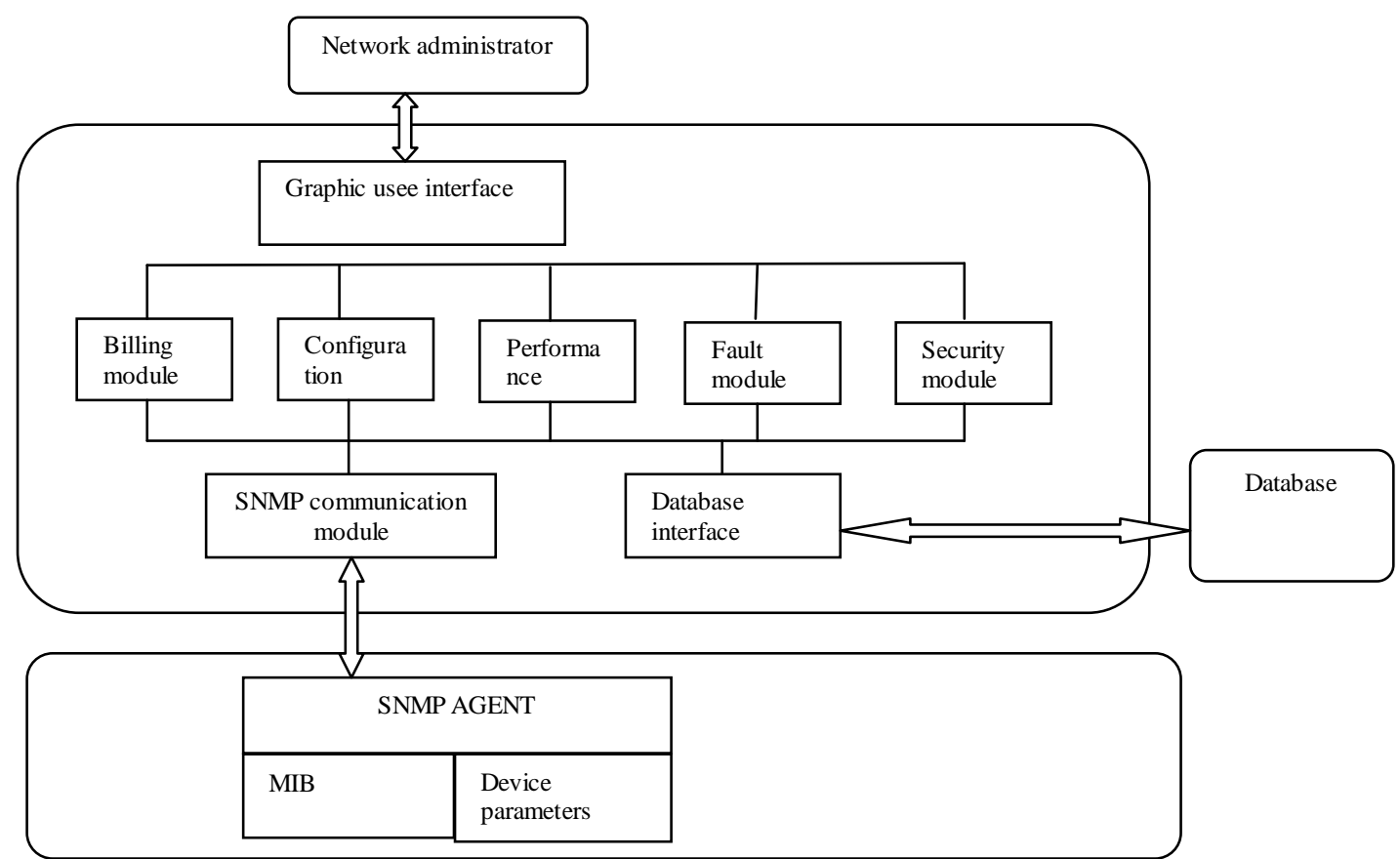

Figure 3.1 Functional Structure Diagram of Computer Network Management System

\section{Security Management Module}

Security management is mainly to ensure that users can securely and reliably access network resources, and manage security information, resource-access services, and encryption processes. This includes features such as user authentication, permission approval, and network access control (firewall). The goal is to control access to network resources in accordance with local security policies to ensure that the network is not compromised (conscious or unconscious) and to ensure that important information is not accessed by unauthorized users.

The security issues in computer network management systems mainly include the following: First, privatization protection of network data is used to prevent data from being acquired by illegal intruders. Second, authorize the management of all uses to prevent unscrupulous people from posting misleading information. Third, strictly control the access rights and control the access rights of network resources. Fourth, release information about security. Fifth, report on the content of security incidents.

Defense against ARP attacks is an important part of network security management. ARP attacks implement ARP spoofing by forging IP addresses and MAC addresses, and can generate a large amount of ARP traffic in the network to block the network. As long as the attacker continuously sends out the fake ARP response packet, the IP-MAC entry in the target host ARP cache can be changed, causing network interruption or man-in-the-middle attack. The ARP alarm system can obtain information and compare it. If a number of different IPs are found to correspond to the unified network card, it is determined that ARP spoofing occurs and the administrator is immediately notified. The process of preventing network ARP attacks is shown in Figure 3.2. 


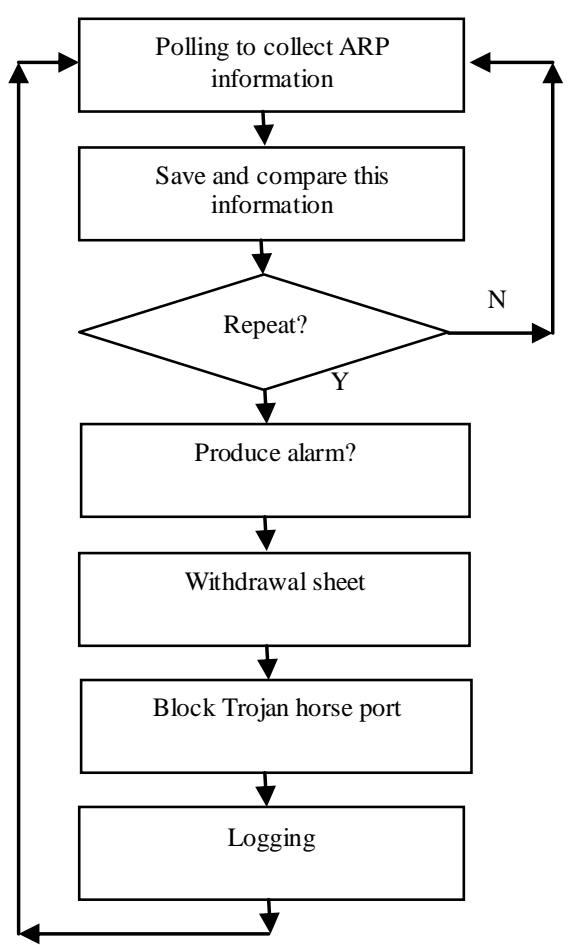

Figure 3.2 ARP Monitoring Diagram

\section{Performance Management Module}

Performance management is the monitoring and tracking of different performance data, scanning the data, and automatically analyzing existing performance data and historical data. Then measure the performance of all aspects of the network. If it is found that the performance is degraded, it is necessary to start the fault management function in time to provide continuous and reliable communication, ensure the quality of service, improve the utilization of network resources, and optimize network resources. Performance management includes performance measurement, performance analysis, and performance management control.

The data acquisition module is the first link in the network performance management process, and is also a vital part of network performance management. It is the basis for subsequent processes such as business processing and data storage. The process of data traffic collection is as follows: 1 . Obtain an SNMP data packet; 2. The service program sends a request message, and the agent receives the request message sent by the service program, and transmits it to the processing module for processing, and sends the response message to the service program; 3 . Encode the data packet according to the rules; 4 . Receive the response message. If the communication is successful and the message is correct, the data analysis module is called. If there is an error, the exception processing is performed. And continuously collect at a sampling frequency of 5 minutes each time. The data collection process is shown in Figure 3.3. 


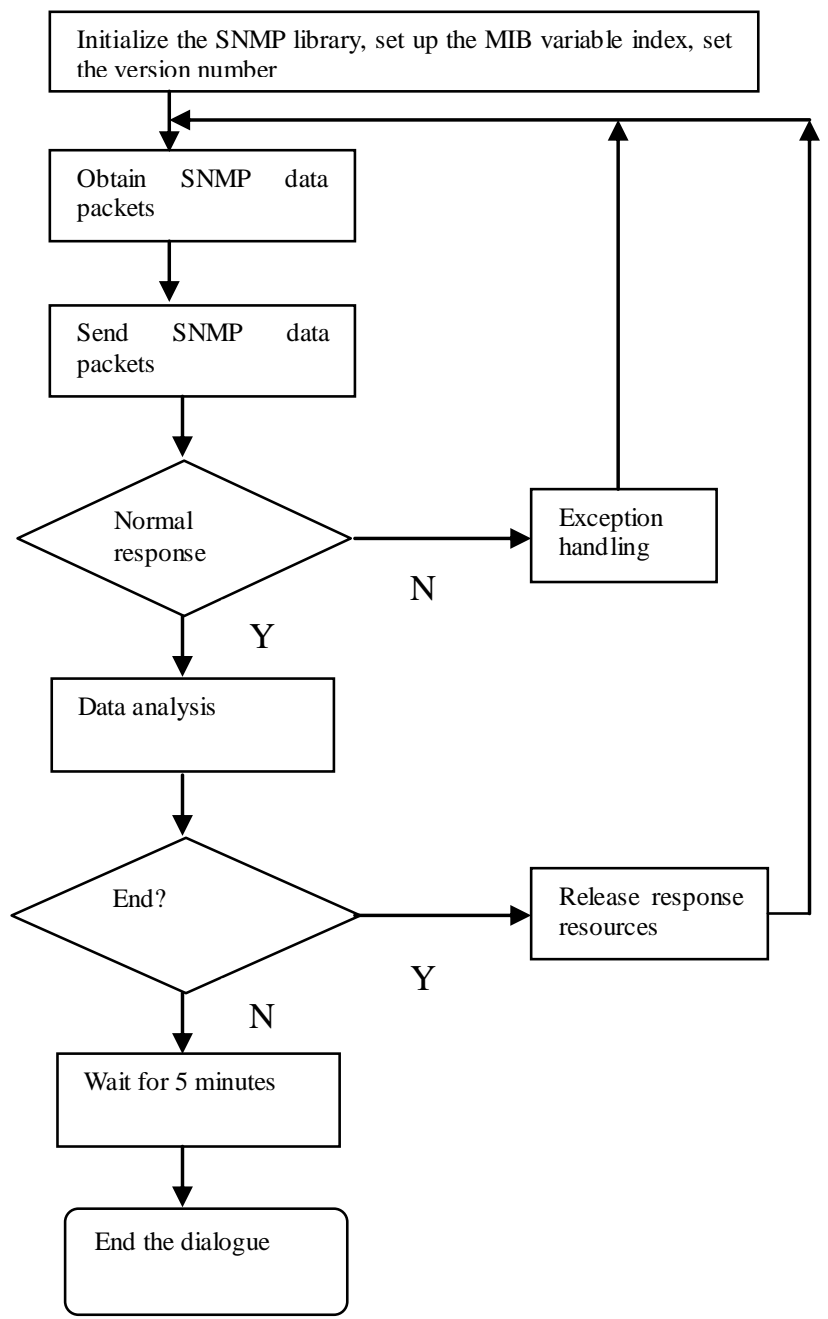

Figure 3.3 Data Collection Diagram

\section{Fault Management Module}

Fault management is the ability to quickly discover, locate, and troubleshoot network failures and dynamically maintain the network. It mainly manages the faults as follows: First, the fault is detected. Maintenance of faults occurring in the network, detection of logs with errors and receipt of reports of faults. Second, troubleshoot the fault. The computer network management system queries the cause of the fault, finds the cause of the fault, and performs a diagnostic test on the fault to determine the exact location of the fault. Third, the computer network management system can correct the fault. The computer network management system searches for the location of the fault, then removes the fault from the system, and finally repairs it according to the cause of the fault.

Fault information alarms need to set thresholds for indicators that affect network performance. By performing threshold detection, you can alert management personnel when performance problems occur in the network. The fault alarm process is shown in Figure 3.2. 


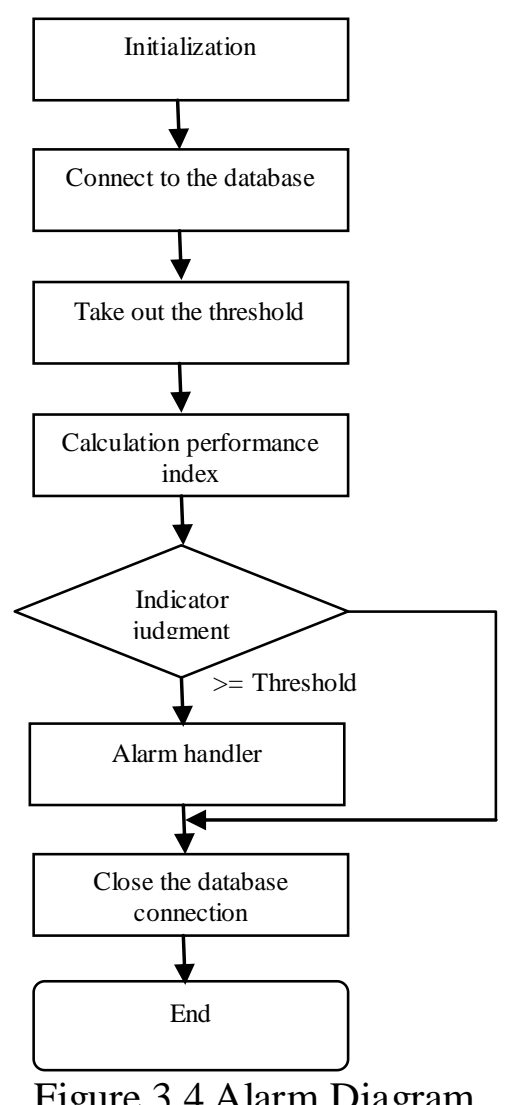

\section{Configuration Management Module}

Configuration management is the most basic network management function, mainly responsible for monitoring and controlling the configuration status of the network. In the process of network establishment, expansion, transformation and operation, the network topology, resource allocation, usage status and other information are defined, monitored and modified, and network redistribution under predetermined conditions. Its main functions include: defining configuration information, setting and modifying attribute values, defining and modifying relationships, initializing and shutting down networks, software distribution, network planning, and resource management. Its purpose is to achieve specific functions to achieve optimal network performance.

\section{Cost Management Module}

Cost management is to record and collect the usage of network resources for the correct calculation and collection of the cost of using the network service by the user, and perform statistics on the utilization of network resources.

\section{Conclusion}

In the context of the development of "big data era", computer networks are facing severe challenges and challenges. Computer network management system is a distributed network application system based on software and hardware. Its purpose is to manage the network, in order to reduce network failures, let the business systems and application systems carried on the network run smoothly. Network management system develops with the development of computer network, which is the inevitable outcome of the development of computer network. The quality of network management will directly affect the quality of network operation. Therefore, the design of computer network management system under the background of big data is very important and necessary.

\section{References}

[1] Research on Computer Network Information Security and Protection Strategy [A]. Zhang Zhe. Decision Forum - Proceedings of the Symposium on the Collaborative Development of the 
Integration of Government, Industry, Education and Research (Part 2) [C]. 2015

[2] Emergence, Computational Science and Big Data: A Summary of the Academic Report of Professor Paul Humphreys and the Chinese Version of His Works[J]. Liu Yiyu, Xue Yonghong, Li Yajuan. Philosophical Analysis. 2018(02)

[3] The Impact of Information Security Issues in the Internet Age on China's Overall National Security and Government Countermeasures [D]. Gu Yan. University of International Business and Economics 2015

[4] Exploration on Dynamic Network Security Defense Strategy of Online Verification Work in Land Change Survey under the Background of "Internet +" [J]. Huang Yan, Xu Weidong, Ha Xueping, Wang Jiandi. Land and Resources Information. 2017(01)

[5] Research on Network Information Security and Protection Strategy Based on the Background of Big Data Era [J]. Zhu Guangjun, Meng Zidong. China New Communication. 2018(02)

[6] Research on Computer Network Information Security Protection Strategy and Evaluation Algorithm [J]. Long Zhenyue, Wei Lihao, Liang Zheheng, Ai Jieqing. Modern Electronic Technology. 2015(23)

[7] Computer Network Information Security Risks and Protective Measures under the Background of Big Data [J]. Liu Mengfei. Modern Industrial Economy and Informatization. 2017(21)

[8] Research on Computer Network Information Management and Security Protection Countermeasures [J]. Li Qun, Ren Tianyu, Wang Xiaohu, Wang Chao, Shi Enjie, Fang Lei. Automation and Instrumentation. 2017(09) 